\title{
Repetitive behavior profiles: Consistency across autism spectrum disorder cohorts and divergence from Prader-Willi syndrome
}

\author{
Cindi G. Flores • Gregory Valcante • Steve Guter • Annette Zaytoun • Emily Wray • \\ Lindsay Bell • Suma Jacob • Mark H. Lewis • Daniel J. Driscoll • Edwin H. Cook Jr. • \\ Soo-Jeong Kim
}

Received: 22 February 2011 / Accepted: 14 August 2011 / Published online: 1 September 2011

(C) Springer Science+Business Media, LLC 2011

\begin{abstract}
Restricted and repetitive behavior (RRB) is a group of heterogeneous maladaptive behaviors. RRB is one of the key diagnostic features of autism spectrum disorders (ASDs) and also commonly observed in Prader-Willi syndrome (PWS). In this study, we assessed RRB using the Repetitive Behavior Scale-Revised (RBS-R) in two ASD samples (University of Illinois at Chicago [UIC] and University of Florida [UF]) and one PWS sample. We compared the RBS-R item endorsements across three ASD cohorts (UIC, UF and an ASD sample from Lam, The Repetitive Behavior Scale-Revised: independent validation and the effect of subject variables, PhD thesis, 2004), and a PWS sample. We also compared the mean RBS-R subscale/
\end{abstract}

C. G. Flores · G. Valcante $\cdot$ A. Zaytoun • E. Wray • L. Bell •

M. H. Lewis $\cdot$ S.-J. Kim

Department of Psychiatry, University of Florida,

Gainesville, FL, USA

S. Guter $\cdot$ S. Jacob $\cdot$ E. H. Cook Jr.

Department of Psychiatry, University of Illinois at Chicago,

Chicago, IL, USA

\section{J. Driscoll}

Department of Pediatrics, University of Florida,

Gainesville, FL, USA

\section{S.-J. Kim}

Department of Psychiatry and Behavioral Sciences,

University of Washington,

Seattle, WA, USA

\section{S.-J. Kim $(\bowtie)$}

Center for Integrative Brain Research,

Seattle Children's Research Institute,

M/S C9S-10, 1900 9th Ave,

Seattle, WA 98101, USA

e-mail:kimsooj@uw.edu sum scores across the UIC, UF and PWS samples; across the combined ASD (UIC+UF), PWS-deletion and PWSdisomy groups; and across the combined ASD sample, PWS subgroup with a Social Communication Questionnaire (SCQ) score $\geq 15$, and PWS subgroup with a SCQ score $<15$. Despite the highly heterogeneous nature, the three ASD samples (UIC, UF and Lam's) showed a similar pattern of the RBS-R endorsements, and the mean RBS-R scores were not different between the UIC and UF samples. However, higher RRB was noted in the ASD sample compared with the PWS sample, as well as in the PWS subgroup with a SCQ score $\geq 15$ compared with the PWS subgroup with a SCQ score $<15$. Study limitations include a small sample size, a wide age range of our participants, and not controlling for potential covariates. A future replication study using a larger sample and further investigation into the genetic bases of overlapping ASD and RRB phenomenology are needed, given the higher RRB in the PWS subgroup with a SCQ score $\geq 15$.

Keywords RRB $\cdot$ ASD $\cdot$ PWS $\cdot$ RBS-R

\section{Introduction}

Restricted and repetitive behavior (RRB) is a group of heterogeneous maladaptive behaviors, such as flapping arms, lining up objects, peculiar fascination with odd objects or parts of objects, a very narrow set of restricted interests, intolerance to changes in routines, and insistence on sameness (Lewis and Kim 2009). RRB is one of the core features of autism spectrum disorders (ASDs), along with deficits in reciprocal social communication (APA 2000). Several family studies in ASDs have suggested genes 
controlling RRB are likely independent of genes controlling social or communication deficits (Silverman et al. 2002; Ronald et al. 2006; Mandy and Skuse 2008).

Although RRB is most frequently associated with ASDs, it is also commonly observed in other neurodevelopmental disorders, such as Prader-Willi syndrome (PWS). PWS is a rare genetic disorder caused by an absence of paternally expressed genes within the chromosome 15q11.2-q13 region via one of three main genetic mechanisms: deletion of paternally expressed genes (65-75\%), maternal uniparental disomy (UPD; 20-30\%) and imprinting defect (ID; 1-3\%) (Cassidy and Driscoll 2009; Horsthemke and Buiting 2008; Nicholls and Knepper 2001). One of the most prominent clinical characteristics of PWS is significant hyperphagia leading to early childhood morbid obesity (Cassidy and Driscoll 2009). Interestingly, studies have reported a high level and wide range of RRB extending beyond food-related behavior among individuals with PWS (Greaves et al. 2006). For instance, skin-picking is reported in $69-100 \%$ of individuals with PWS (Butler et al. 2004; Torrado et al. 2006; Dykens et al. 1999). Prominent obsessive compulsive symptoms (hoarding, ordering/ arranging, concerns with symmetry/exactness, rewriting, need to tell/know/ask) were also reported in $37-58 \%$ of individuals with PWS (Dykens et al. 1996). A recent study (Greaves et al. 2006) also suggested a similar level of RRB among children with ASDs and children with PWS using the Childhood Routines Inventory (CRI) (Evans et al. 1997). In addition, an increased rate of ASDs has been reported among individuals with PWS, especially among those with maternal UPD compared to those with a deletion $(37.7 \%$ vs. $18.6 \%$ ) (Veltman et al. 2005). However, few studies have examined the RRB phenomenology among individuals with PWS using a comprehensive RRB measure, nor examined the characteristics of RRB across these two populations, despite the reports of increased rates of RRB as well as ASDs among individuals with PWS.

Despite the heterogeneous nature of RRB, specific forms of RRB may be characterized and quantified using a comprehensive behavioral measure, such as the Repetitive Behavior Scale-Revised (RBS-R). The RBS-R is an empirically derived, standardized, and psychometrically sound rating scale used to measure various RRB among individuals with developmental disorders (Bodfish et al. 1995, 2000). The RBS-R includes 43 items grouped into six empirically derived subscales: Stereotyped, Self-injurious, Compulsive, Ritualistic, Sameness, and Restricted behaviors. The RBS-R has been used extensively in ASDs to characterize specific forms of RRB across a wide age range (Bodfish et al. 2000; Mirenda et al. 2010; Lam and Aman 2007; Lam 2004). For instance, Lam examined the RBS-R data from 307 individuals with ASDs between the ages of 3 and
48 years, and found the RBS-R individual item endorsement rates ranging from $17.3 \%$ to $80.4 \%$ of the sample (Lam 2004).

In the present study, we obtained the RBS-R data from two ASD samples (University of Illinois at Chicago [UIC] and University of Florida [UF]) and one PWS sample. The primary aim was to compare the RBS-R item endorsements in these two ASD cohorts (UIC and UF), and to compare them with Lam's (2004) findings. The secondary aim was to compare the RRB characteristics between ASD and PWS samples, given the reports of increased rates of RRB as well as ASDs among individuals with PWS.

Since PWS may be divided into two genetically distinct subgroups, i.e., a PWS-deletion subgroup (i.e., PWS due to deletion) vs. a PWS-disomy subgroup (PWS due to either UPD or ID), we also compared the RRB characteristics between these two PWS subgroups. Additionally, because of increased rates of ASDs among individuals with PWS, we divided PWS group into two subgroups by the Social Communication Questionnaire (SCQ) total score (Rutter et al. 2003). Since the SCQ total score $\geq 15$ has been shown to indicate high probability of ASDs (Rutter et al. 2003; Chandler et al. 2007; Corsello et al. 2007; Charman et al. 2007; Bishop and Norbury 2002), we compared the RBS-R subscale/sum scores across three subgroups: PWS with the SCQ total score $<15$, PWS with the SCQ total score $\geq 15$, and the combined ASD sample (UIC+UF).

\section{Methods}

Sample characteristics

This study was approved by the UIC and UF Institutional Review Boards. All participants were provided with a description of the study prior to obtaining informed consent. For this report, the inclusion criteria for the UIC sample include meeting ASD or autism classification on both Autism Diagnostic Interview-Revised (ADI-R) (Lord et al. 1994) and Autism Diagnostic Observation Schedule (ADOS) (Lord et al. 2000) along with a best estimate diagnosis of an ASD (i.e., autistic disorder, Asperger disorder, or pervasive developmental disorder-not otherwise specified) according to the DSM-IV-TR criteria (APA 2000). The UIC sample consisted of 103 probands (M/F= $87: 16$ ) with a mean age of $9 \pm 5$ years, 10 months (range: 3 years, 2 months to 33 years, 10 months); $63.1 \%$ Caucasian; $6.8 \%$ on psychotropic medications; and $71.8 \%$ classified as "strictly defined autism (autism classification on both ADI-R and ADOS)." There were 12 missing RBS-R data points with a completion rate of $99.7 \%$.

The inclusion criteria for the UF sample include chronological age between 6 and 18 years, clinical diagnosis of an 
ASD, and an absence of a genetic diagnosis. The UF sample did not receive ADI-R or ADOS evaluations, because they were recruited for a mail survey study. For this report, therefore, we used the SCQ total score to exclude those who scored below 15 , as previous studies have suggested using a cutoff score of 15 to differentiate children with ASDs from children without ASDs (Rutter et al. 2003; Chandler et al. 2007; Corsello et al. 2007; Charman et al. 2007; Bishop and Norbury 2002). The UF sample consisted of 104 individuals $(\mathrm{M} / \mathrm{F}=83: 21)$ with a mean age of 10 years, 9 months \pm 3 years, 6 months (range: 5 years, 5 months to 18 years, 5 months); $76.0 \%$ Caucasian; and $61.5 \%$ on psychotropic medications. There were no missing RBS-R data.

Lam's (2004) sample was also recruited through a mail survey study. The sample consisted of 307 individuals with a clinical diagnosis of an ASD with a mean age of 15 years, 4 months \pm 9 years, 7 months (range: 3 to 48 years); $82.4 \%$ male; $69.1 \%$ Caucasian; $81.4 \%$ autistic disorder; $40.3 \%$ with mild to profound intellectual disability; $53.4 \%$ on psychotropic medications.

The inclusion criteria for the PWS sample included a genetically confirmed diagnosis of PWS and a chronological age of at least 3.0 years. The PWS participants were recruited mainly from another PWS study at UF or through word of mouth. The probands were not prescreened for having a comorbid ASD diagnosis; however, we administered the SCQ as a part of the assessment. The PWS sample consisted of 45 individuals $(\mathrm{M} / \mathrm{F}=20: 25)$ with a mean age of 10 years, 7 months \pm 8 years, 7 months (range: 3 to 37 years); $91.1 \%$ Caucasian; $55.6 \%$ (25/45) with PWS-deletion vs. $44.4 \%$ (20/45) with PWS-disomy (i.e., maternal UPD or ID). The SCQ scores were available for 44 PWS participants with a mean total score of $10.0 \pm 5.6$ in the PWSdeletion subgroup $(n=24)$ and $12.0 \pm 6.6$ in the PWSdisomy subgroup $(n=20)$. A total of 12 participants with PWS scored $\geq 15$ on the SCQ; $16.7 \%(5 / 24)$ of participants in the PWS-deletion subgroup and 35.0\% (7/20) in the PWS-disomy subgroup scored $\geq 15$ on the SCQ. There were no missing RBS-R data.

\section{Statistical methods}

RBS-R endorsement rate refers to a percentage of individuals for whom a particular item was endorsed. A $\chi^{2}$ test was used to compare the RBS-R item endorsement rates between the UIC and UF datasets. Because of a small and unbalanced sample size in the PWS sample, Fisher's exact test was used to compare the RBS-R item endorsements between the combined ASD (UIC+UF) and PWS samples, and between PWS-deletion and PWS-disomy subgroups. Fisher's exact test was used to compare the percentage of individuals with the SCQ total score $\geq 15$ between PWS-deletion and PWSdisomy subgroups. The General Linear Model (GLM) as implemented in IBM ${ }^{\circledR}$ SPSS ${ }^{\circledR}$ Statistics package (version 19), was used to compare the means of the RBS-R subscale and sum scores across the UIC, UF, and PWS samples, across the combined ASD sample, PWS-deletion, and PWS-disomy subgroups, and across the PWS subgroup with a lower SCQ score $(<15)$, PWS subgroup with a higher SCQ score $(\geq 15)$, and the combined ASD sample. Age and gender were treated as covariates for all GLM analyses. Bonferroni's test was used to identify significant group differences with a post-hoc value of $p<0.05$. The significance for the $\chi^{2}$ test, Fisher's exact test and GLM was set at a value of $p<0.001$ to correct for multiple comparisons.

\section{Results}

The RBS-R individual item endorsements were 15.9$87.4 \%$ in the combined ASD samples (UIC+UF) and 4.4 $64.4 \%$ in the PWS sample. Figure 1 shows the pattern of RBS-R individual item endorsements across three ASD cohorts (UIC, UF and Lam's samples) and a PWS sample. Figure 2 represents the pattern of RBS-R individual item endorsements across combined ASD (UIC+UF), PWSdeletion and PWS-disomy groups. Interestingly, all three ASD cohorts (UIC, UF, and Lam) showed a similar pattern of the RBS-R individual item endorsements compared with the PWS sample. A $\chi^{2}$ test revealed no statistically significant differences in the RBS- $R$ individual item endorsements between UIC and UF samples; however, the item 14 "skin picking" showed a trend for a higher endorsement in the UF sample than in the UIC sample $\left(\chi^{2}=6.68, d f=1, p=0.010\right)$.

Table 1 shows the endorsement rates of all RBS-R individual items between the combined ASD and PWS samples, and between PWS-deletion and PWS-disomy subgroups. Overall, all but two RBS-R individual items (items 14 and 15) were more frequently endorsed in the ASD sample than in the PWS sample. Among those, 16 items $(3-8,30,32,34,36,40-43)$ from four RBS-R subscales (Stereotyped/Self-injurious/Sameness/Restricted behaviors) showed statistically significant differences between the ASD and PWS samples. Neither of the two items (item 14 and 15) endorsed more frequently in the PWS sample than in the ASD sample showed statistically significant differences (item $14, \chi^{2}=5.827, d f=1, p=$ 0.020 ; item $15, \chi^{2}=0.084, d f=1, p=0.866$ ), although the item 14 "skin-picking" showed a trend for higher frequency in the PWS sample than in the ASD sample (Table 1). The PWS-deletion and PWS-disomy subgroups did not show statistically significant group differences in the RBS-R individual item endorsement; however, the PWS-deletion subgroup showed a trend for higher endorsements of four items from two RBS-R subscale scores (Self-injurious/ 


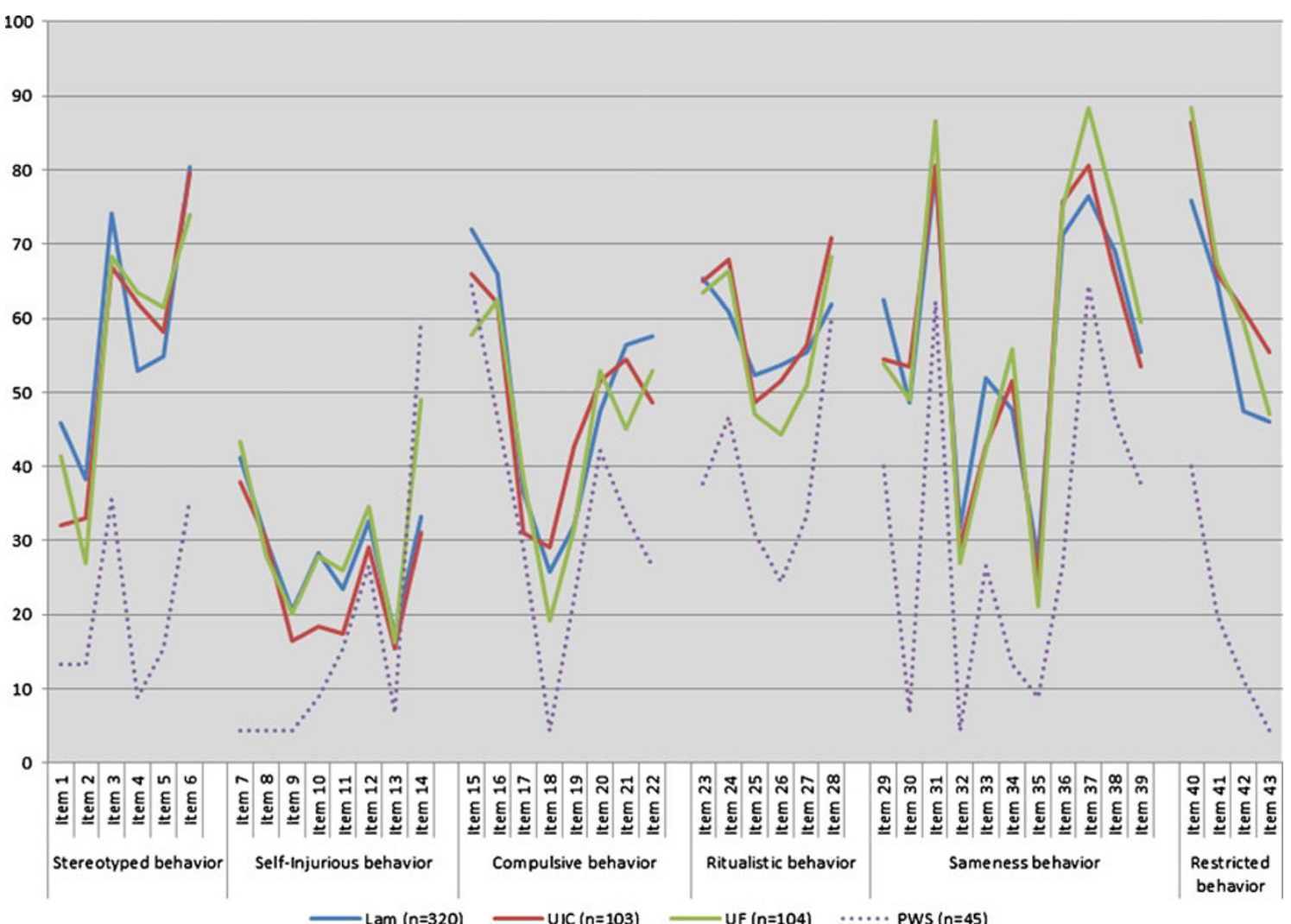

Fig. 1 The RBS-R individual item endorsement rates across three ASD cohorts and one PWS sample

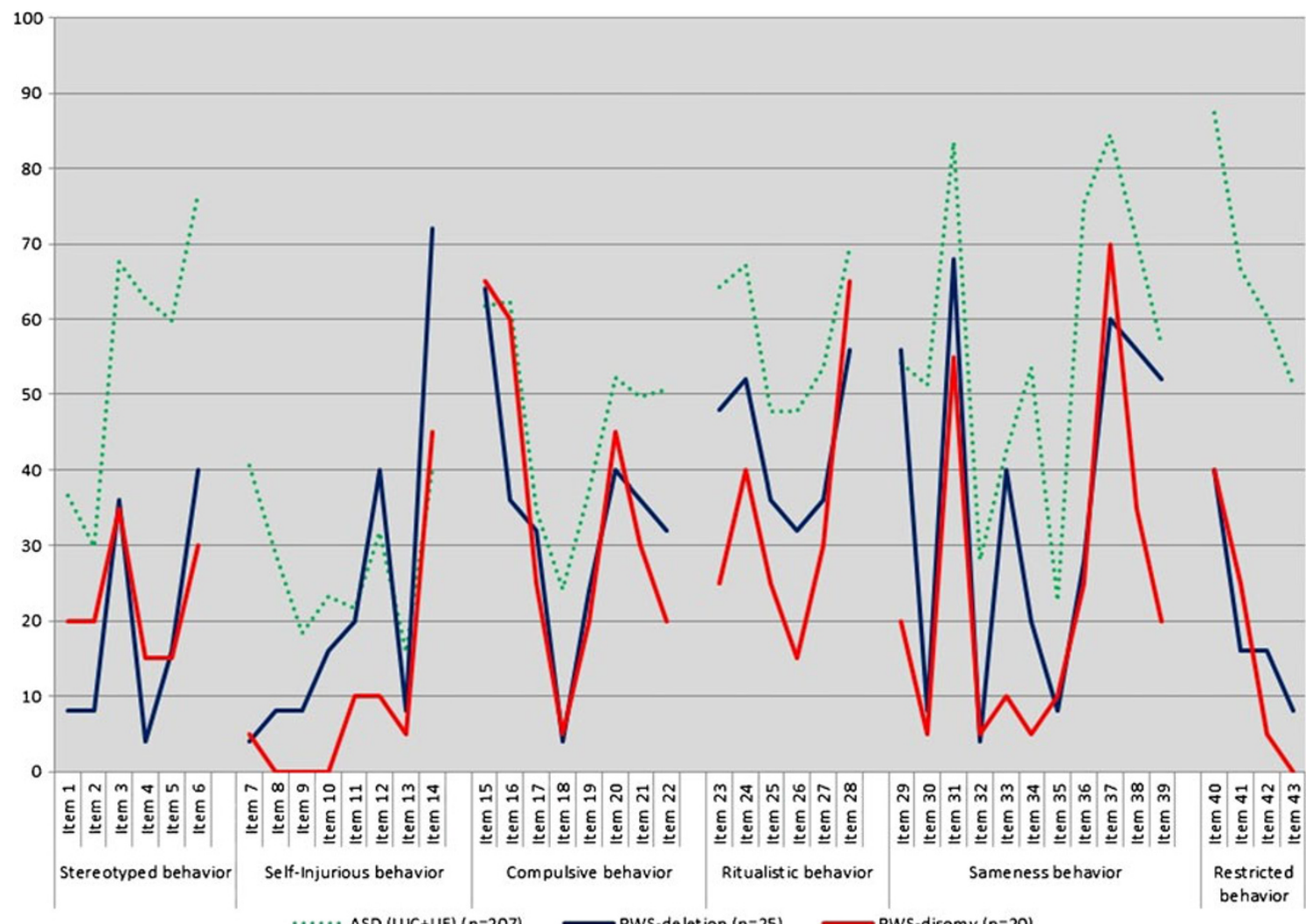

Fig. 2 The RBS-R individual item endorsement rates across a combined ASD sample, a PWS-deletion subgroup and a PWS-disomy subgroup 
Table 1 Comparison frequency of RBS-R individual endorsements across different samples

\begin{tabular}{|c|c|c|c|c|c|c|c|c|c|c|}
\hline & RBS-R item & Description & $\begin{array}{l}\text { ASD } \\
(n=207)\end{array}$ & $\begin{array}{l}\text { PWS } \\
(n=45)\end{array}$ & $\chi^{2}(d f=1)$ & $p$ & $\begin{array}{l}\text { PWS-deletion } \\
(n=25)\end{array}$ & $\begin{array}{l}\text { PWS-disomy } \\
(n=20)\end{array}$ & $\chi^{2}(d f=1)$ & $p$ \\
\hline \multirow[t]{6}{*}{ Stereotyped behavior } & 01 & Body & 36.7 & 13.3 & 9.206 & 0.003 & 8 & 20 & 1.385 & $\mathrm{~ns}$ \\
\hline & 02 & Head & 30.0 & 13.3 & 5.181 & 0.026 & 8 & 20 & 1.385 & ns \\
\hline & 03 & Finger & 67.6 & 35.6 & 16.487 & $<0.001$ & 36 & 35 & 0.005 & ns \\
\hline & 04 & Locomotion & 62.8 & 8.9 & 44.111 & $<0.001$ & 4 & 15 & 1.660 & ns \\
\hline & 05 & Object & 59.9 & 15.6 & 29.125 & $<0.001$ & 16 & 15 & 0.008 & ns \\
\hline & 06 & Sensory & 76.8 & 35.6 & 29.650 & $<0.001$ & 40 & 30 & 0.485 & ns \\
\hline \multirow[t]{8}{*}{ Self-injurious behavior } & 07 & Hit body & 40.6 & 4.4 & 21.470 & $<0.001$ & 4 & 5 & 0.026 & ns \\
\hline & 08 & Hit surface & 29.0 & 4.4 & 12.001 & $<0.001$ & 8 & 0 & 1.674 & $\mathrm{~ns}$ \\
\hline & 09 & hit object & 18.4 & 4.4 & 5.358 & 0.023 & 8 & 0 & 1.674 & $\mathrm{~ns}$ \\
\hline & 10 & bite self & 23.2 & 8.9 & 4.615 & 0.040 & 16 & 0 & 3.512 & $\mathrm{~ns}$ \\
\hline & 11 & Pull hair/skin & 21.7 & 15.6 & 0.863 & ns & 20 & 10 & 0.846 & $\mathrm{~ns}$ \\
\hline & 12 & Scratch & 31.9 & 26.7 & 0.471 & ns & 40 & 10 & 5.114 & 0.040 \\
\hline & 13 & Inserts & 15.9 & 6.7 & 2.597 & $\mathrm{~ns}$ & 8 & 5 & 0.161 & ns \\
\hline & 14 & Picks skin & 40.1 & 60.0 & 5.827 & 0.020 & 72 & 45 & 3.375 & $\mathrm{~ns}$ \\
\hline \multirow[t]{8}{*}{ Compulsive behavior } & 15 & Order & 61.8 & 64.4 & 0.084 & ns & 64 & 65 & 0.005 & $\mathrm{~ns}$ \\
\hline & 16 & Complete & 62.3 & 46.7 & 3.759 & 0.065 & 36 & 60 & 2.571 & $\mathrm{~ns}$ \\
\hline & 17 & Wash & 34.8 & 28.9 & 0.606 & ns & 32 & 25 & 0.265 & ns \\
\hline & 18 & Check & 24.2 & 4.4 & 8.911 & 0.002 & 4 & 5 & 0.026 & ns \\
\hline & 19 & Count & 37.2 & 22.2 & 3.667 & 0.059 & 24 & 20 & 0.103 & ns \\
\hline & 20 & Hoard & 52.2 & 42.2 & 1.464 & ns & 40 & 45 & 0.114 & ns \\
\hline & 21 & Repeat & 49.8 & 33.3 & 4.118 & 0.048 & 36 & 30 & 0.180 & $\mathrm{~ns}$ \\
\hline & 22 & Touch/Tap & 50.7 & 26.7 & 8.602 & 0.005 & 32 & 20 & 0.818 & ns \\
\hline \multirow[t]{6}{*}{ Ritualistic behavior } & 23 & Eating & 64.3 & 37.8 & 10.753 & 0.001 & 48 & 25 & 2.501 & ns \\
\hline & 24 & Sleeping & 67.1 & 46.7 & 6.691 & 0.016 & 52 & 40 & 0.643 & ns \\
\hline & 25 & Self care & 47.8 & 31.1 & 4.175 & 0.048 & 36 & 25 & 0.627 & ns \\
\hline & 26 & Transportation & 47.8 & 24.4 & 8.216 & 0.005 & 32 & 15 & 1.739 & ns \\
\hline & 27 & Play/leisure & 53.6 & 33.3 & 6.087 & 0.021 & 36 & 30 & 0.180 & $\mathrm{~ns}$ \\
\hline & 28 & Communication & 69.6 & 60.0 & 1.668 & ns & 56 & 65 & 0.375 & ns \\
\hline \multirow[t]{11}{*}{ Sameness behavior } & 29 & Object & 54.1 & 40.0 & 2.945 & ns & 56 & 20 & 6.000 & 0.018 \\
\hline & 30 & Place & 51.2 & 6.7 & 29.877 & $<0.001$ & 8 & 5 & 0.161 & ns \\
\hline & 31 & Interruption & 83.6 & 62.2 & 10.440 & 0.003 & 68 & 55 & 0.799 & $\mathrm{~ns}$ \\
\hline & 32 & walking & 28.0 & 4.4 & 11.325 & $<0.001$ & 4 & 5 & 0.026 & ns \\
\hline & 33 & Sitting & 42.5 & 26.7 & 3.877 & 0.064 & 40 & 10 & 5.114 & 0.040 \\
\hline & 34 & Appearance & 53.6 & 13.3 & 24.124 & $<0.001$ & 20 & 5 & 2.163 & ns \\
\hline & 35 & door & 22.7 & 8.9 & 4.371 & 0.040 & 8 & 10 & 0.055 & ns \\
\hline & 36 & videotapes & 75.4 & 26.7 & 39.443 & $<0.001$ & 28 & 25 & 0.051 & ns \\
\hline & 37 & transition & 84.5 & 64.4 & 9.682 & 0.003 & 60 & 70 & 0.485 & ns \\
\hline & 38 & routine & 70.5 & 46.7 & 9.418 & 0.003 & 56 & 35 & 1.969 & $\mathrm{~ns}$ \\
\hline & 39 & time & 56.5 & 37.8 & 5.368 & 0.022 & 52 & 20 & 4.840 & 0.035 \\
\hline \multirow[t]{4}{*}{ Restricted behavior } & 40 & Preoccupation & 87.4 & 40.0 & 50.088 & $<0.001$ & 40 & 40 & 0.001 & ns \\
\hline & 41 & attachment & 66.7 & 20.0 & 33.606 & $<0.001$ & 16 & 25 & 0.563 & ns \\
\hline & 42 & Part of object & 60.4 & 11.1 & 35.937 & $<0.001$ & 16 & 5 & 1.361 & ns \\
\hline & 43 & movement & 51.2 & 4.4 & 33.007 & $<0.001$ & 8 & 0 & 1.674 & ns \\
\hline
\end{tabular}

Significant $p$ values (less than 0.001 ) are presented in bold typeface. "ns" indicates any p values higher than 0.1

Sameness behaviors); "rubs or scratches self" (item 12, $\chi^{2}=$ 5.114, $d f=1, p=0.040$ ), "insists that things remain in the same places" (item $29, \chi^{2}=6.000, d f=1, p=0.018$ ), "insists on sitting at the same place" (item $33, \chi^{2}=5.114, d f=1, p=$ 0.040 ), and "insists that specific things take place at specific times" (item $39, \chi^{2}=4.840, d f=1, p=0.035$ ). 
Significant group differences $(p<0.001)$ were observed for five RBS-R scores including Stereotyped, Ritualistic, Sameness, Restricted behaviors and Sum among the UIC, UF and PWS samples (Table 2). Bonferroni's test revealed that significant group differences (post-hoc $p<0.05$ ) were between the UIC and PWS samples, and between the UF and PWS samples, but not between the two ASD samples. When we examined the same RBS-R scores among combined ASD, PWS-deletion and PWS-disomy, significant group differences existed between ASD and either PWS group for the same five RBS-R scores (Stereotyped/ Ritualistic/Sameness/Restricted/Sum, $p<0.001$ ) (Table 3).

No significant group difference was observed in the percentage of individuals whose SCQ total score $\geq 15$ between the PWS-deletion and PWS-disomy subgroups $\left(\chi^{2}=1.104, d f=1, p=0.329\right)$. When the RBS-R scores were compared across the combined ASD sample, the PWS subgroup with a lower SCQ score $(<15)$, and the PWS subgroup with a higher SCQ score $(\geq 15)$; statistically significant group differences were observed between the PWS subgroup with a lower SCQ score and the PWS subgroup with a higher SCQ score, and between the PWS subgroup with a lower SCQ score and combined ASD groups in five RBS-R scores including Stereotyped, Compulsive, Ritualistic, Sameness behaviors and Sum score $(p<0.001)$ (Table 4). The self-injurious behavior score was not different across these three groups, and the restricted behavior score was significantly lower in both PWS groups than in the combined ASD sample. The effect of covariates, age and gender, were not significant except for the age effect on Stereotyped behavior in the ASD sample - less severe Stereotyped behavior was endorsed as age increased.

\section{Discussion}

To our knowledge, this is the first study that directly compared the characteristics of RRB measured on the RBS$\mathrm{R}$ across different ASD cohorts or between ASD and PWS samples. Despite the highly heterogeneous nature of RRB, the three ASD samples (UIC, UF and Lam's) showed a similar pattern of RBS-R individual item endorsements (Fig. 1). In addition, mean subscale scores of the RBS-R were not different between two ASD datasets (UIC and UF), even though substantial differences existed between these cohorts in terms of recruitment and ascertainment protocols, geographical location (Illinois vs. Florida), and the rate of concurrent psychotropic medication use.

Contrary to the recent study (Greaves et al. 2006) that reported a similar level of RRB measured on the CRI between ASD and PWS, we observed significantly less frequent and less severe RRB in the PWS sample compared with the ASD sample in this study. This may be because the RBS-R and CRI measure different aspects of RRB; for instance, the RBS-R yields six empirically derived factors (Stereotyped, Self-injurious, Compulsive, Ritualistic, Sameness and Restricted Behaviors), while the CRI yields two factors ("just right" and "repetitive behavior"). In addition, $\mathrm{RRB}$ is one of the diagnostic features of ASDs; therefore, individuals with ASDs are expected to have higher level of RRB than those without ASDs.

This study also made several interesting observations. First, a trend of higher "skin-picking" was revealed in the PWS sample compared with the ASD sample, consistent with previous reports of high skin-picking behaviors among individuals with PWS (Butler et al. 2004; Torrado et al.

Table 2 Comparison of means of RBS-R subscale and sum scores across two ASD and one PWS samples after controlling for covariates (age and gender)

\begin{tabular}{|c|c|c|c|c|c|c|}
\hline & UIC $(n=103)$ EMM (SE) & UF $(n=104)$ EMM (SE) & PWS $(n=45)$ EMM (SE) & GLM $F_{2,247}$ & $p$ & Post-hoc $(p)$ \\
\hline Stereotyped behavior & $5.9(0.4)$ & $5.6(0.4)$ & $2.1(0.6)$ & 17.053 & $<0.001$ & $\begin{array}{l}\text { UIC vs. PWS } * * * \\
\text { UF vs. PWS*** }\end{array}$ \\
\hline Self-injurious behavior & $3.3(0.4)$ & $4.2(0.4)$ & $1.9(0.7)$ & 4.105 & 0.018 & \\
\hline Compulsive behavior & $7.1(0.5)$ & $6.3(0.5)$ & $4.2(0.8)$ & 4.162 & 0.017 & \\
\hline Ritualistic behavior & $7.2(0.5)$ & $6.2(0.5)$ & $3.4(0.7)$ & 9.137 & $<0.001$ & $\begin{array}{l}\text { UIC vs. PWS*** } \\
\text { UF vs. PWS** }\end{array}$ \\
\hline Sameness behavior & $11.7(0.7)$ & $11.2(0.7)$ & $4.6(1.1)$ & 14.244 & $<0.001$ & $\begin{array}{l}\text { UIC vs. PWS }{ }^{* * *} \\
\text { UF vs. PWS*** }\end{array}$ \\
\hline Restricted behavior & $5.5(0.3)$ & $4.9(0.3)$ & $1.3(0.5)$ & 26.357 & $<0.001$ & $\begin{array}{l}\text { UIC vs. PWS*** } \\
\text { UF vs. PWS*** }\end{array}$ \\
\hline Sum & $40.7(2.2)$ & $38.4(2.2)$ & $17.5(3.5)$ & 16.262 & $<0.001$ & $\begin{array}{l}\text { UIC vs. PWS } * * * \\
\text { UF vs. PWS*** }\end{array}$ \\
\hline
\end{tabular}

$E M M(S E)$ Estimated marginal means (standard error). Only those with significant differences ( $p \leq 0.001$, presented in bold typeface) were listed in the post-hoc test column and Bonferroni's test was used as a post-hoc procedure

Significance for the post-hoc test was set as $p<0.05$. ${ }^{* *} p<0.01,{ }^{* * *} p<0.001$ 
Table 3 Comparison of means of RBS-R subscale and sum scores across combined ASD, PWS-deletion and PWS-disomy samples after controlling for covariates (age and gender)

\begin{tabular}{|c|c|c|c|c|c|c|}
\hline & $\begin{array}{l}\operatorname{ASD}(n=207) \\
\operatorname{EMM}(\mathrm{SE})\end{array}$ & $\begin{array}{l}\text { PWS-deletion }(n=25) \\
\text { EMM (SE) }\end{array}$ & $\begin{array}{l}\text { PWS-disomy }(n=20) \\
\text { EMM (SE) }\end{array}$ & GLM $F_{2,247}$ & $p$ & Post-hoc $(p)$ \\
\hline Stereotyped behavior & $5.7(0.3)$ & $1.7(0.7)$ & $2.5(0.8)$ & 17.238 & $<0.001$ & $\begin{array}{l}\text { ASD vs. PWS-deletion*** } \\
\text { ASD vs. PWS-disomy** }\end{array}$ \\
\hline Self-injurious behavior & $3.8(0.3)$ & $2.6(0.9)$ & $1(1.1)$ & 3.759 & 0.025 & \\
\hline Compulsive behavior & $6.7(0.4)$ & $4.1(1.1)$ & $4.3(1.2)$ & 3.579 & 0.029 & \\
\hline Ritualistic behavior & $6.7(0.3)$ & $3.7(1)$ & $3(1.1)$ & 8.033 & $<0.001$ & $\begin{array}{l}\text { ASD vs. PWS-deletion* } \\
\text { ASD vs. PWS-disomy** }\end{array}$ \\
\hline Sameness behavior & $11.4(0.5)$ & $5.8(1.5)$ & $3.1(1.7)$ & 14.98 & $<0.001$ & $\begin{array}{l}\text { ASD vs. PWS-deletion** } \\
\text { ASD vs. PWS-disomy*** }\end{array}$ \\
\hline Restricted behavior & $5.2(0.2)$ & $1.3(0.6)$ & $1.3(0.7)$ & 25.203 & $<0.001$ & $\begin{array}{l}\text { ASD vs. PWS-deletion*** } \\
\text { ASD vs. PWS-disomy*** }\end{array}$ \\
\hline Sum & $39.5(1.6)$ & $19.3(4.6)$ & $15.2(5.1)$ & 16.172 & $<0.001$ & $\begin{array}{l}\text { ASD vs. PWS-deletion*** } \\
\text { ASD vs. PWS-disomy }^{* * *}\end{array}$ \\
\hline
\end{tabular}

$E M M(S E)$ estimated marginal means (standard error). Only those with significant differences ( $p \leq 0.001$, presented in bold typeface) were listed in the post-hoc test column and Bonferroni's test was used as a post-hoc procedure

The significance for the post-hoc test was set as $p<0.05$. ${ }^{*} p<0.05,{ }^{* *} p<0.01,{ }^{* * *} p<0.001$

2006; Dykens et al. 1999). Secondly, we found trends of higher endorsements of the RBS-R items related to Selfinjurious and Sameness behaviors as well as higher mean scores of Self-injurious and Sameness behaviors in the PWS-deletion subgroup compared with the PWS-disomy subgroup. This finding may be consistent with previous reports of higher level of RRB in the PWS-deletion subgroup compared with the PWS-disomy subgroup (Torrado et al. 2006; Dykens et al. 1999). However, other RBS-R items or subscale scores did not show a similar pattern in this study. Since specific forms of RRB may be more common among individuals with a specific genetic subtype (i.e., deletion vs. disomy), a replication study in a larger sample set would be worthwhile. Thirdly, we did not find an age or

Table 4 Comparison of RBS-R scores across PWS group with SCQ $<15$, PWS group with SCQ $\geq 15$ and ASD sample after controlling for covariates (age and gender)

\begin{tabular}{|c|c|c|c|c|c|c|}
\hline & $\begin{array}{l}\text { PWS with } \mathrm{SCQ}<15 \\
\text { (L) }(n=32) \\
\text { EMM (SE) }\end{array}$ & $\begin{array}{l}\text { PWS with } S C Q \geq 15 \\
(\mathrm{H})(n=12) \\
\text { EMM (SE) }\end{array}$ & $\begin{array}{l}\text { Combined ASD } \\
(n=207)\end{array}$ & GLM $F_{2,246}$ & $p$ & Post-hoc $(p)$ \\
\hline Stereotyped & $0.9(0.7)$ & $5.1(1.0)$ & $5.7(0.2)$ & 22.474 & $<0.001$ & $\begin{array}{l}\mathrm{L} \text { vs. } \mathrm{H}^{* *} \\
\mathrm{~L} \text { vs. } \mathrm{ASD}^{* * *}\end{array}$ \\
\hline Self-injurious & $1.5(0.8)$ & $2.4(1.3)$ & $3.8(0.3)$ & 3.617 & 0.028 & \\
\hline Compulsive & $2.7(1.0)$ & $8.2(1.5)$ & $6.7(0.4)$ & 8.119 & $<0.001$ & $\begin{array}{l}\text { L vs. } \mathrm{H}^{* *} \\
\mathrm{~L} \text { vs. } \mathrm{ASD}^{* *}\end{array}$ \\
\hline Ritualistic & $2.4(0.9)$ & $6.4(1.4)$ & $6.7(0.3)$ & 10.664 & $<0.001$ & $\begin{array}{l}\text { L vs. } \mathrm{H}^{*} \\
\mathrm{~L} \text { vs. } \mathrm{ASD}^{* * *}\end{array}$ \\
\hline Sameness & $2.4(1.3)$ & $9.4(2.1)$ & $11.4(0.5)$ & 19.479 & $<0.010$ & $\begin{array}{l}\mathrm{L} \text { vs. } \mathrm{H}^{*} \\
\mathrm{~L} \text { vs. } \mathrm{ASD}^{* * *}\end{array}$ \\
\hline Restricted & $0.9(0.6)$ & $2.6(0.9)$ & $5.2(0.2)$ & 25.717 & $<0.010$ & $\begin{array}{l}\text { L vs. ASD }{ }^{* * *} \\
\text { H vs. ASD* }\end{array}$ \\
\hline Sum & $10.9(4.1)$ & $34.1(6.4)$ & $39.6(1.5)$ & 21.257 & $<0.001$ & $\begin{array}{l}\text { L vs. } \mathrm{H}^{* *} \\
\mathrm{~L} \text { vs. ASD*** }\end{array}$ \\
\hline
\end{tabular}

Only those with significant differences ( $p \leq 0.001$, presented in bold typeface) were listed in the post-hoc test column and Bonferroni's test was used as a post-hoc procedure

$L$ PWS group with lower total SCQ score $(<15), H$ PWS group with higher total SCQ score $(\geq 15), E M M$ (SE) estimated marginal means (standard error) Significance for the post-hoc test was set as $p<0.05 .{ }^{*} p<0.05, * * p<0.01,{ }^{* * *} p<0.001$ 
gender effect on the RBS-R except there was a reverse relationship between age and Stereotyped behavior in the ASD sample - less severe Stereotyped behavior was endorsed as age increased. It is not clear why we did not see a similar age effect in the PWS sample. However, it is possible that our PWS sample may not have enough power to detect the difference due to a small sample size; or this may have been because younger participants with PWS may have received early interventions (as they may have been diagnosed at a much younger age, e.g., at birth, due to improvements in genetic diagnostic procedure for PWS), whereas older participants with PWS may have not received appropriate early interventions due to a delayed genetic diagnosis. Fourthly, approximately $16 \%$ of PWSdeletion and $35 \%$ of PWS-disomy subgroups scored $\geq 15$ on the SCQ, consistent with previous reports of increased rates of comorbid ASDs among individuals with PWS (Veltman et al. 2004, 2005; Dimitropoulos and Schultz 2007). The difference in the proportion of individuals with the higher SCQ total score was not statistically significant between PWS-deletion (16.7\%) and PWS-disomy (35.0\%) subgroups. However, this result is still interesting, given the previous reports of higher prevalence of ASDs in PWS with maternal UPD subtype compared with PWS with deletion subtype (Veltman et al. 2004; Milner et al. 2005) as well as reports of an association between maternal interstitial duplication of the 15q11-q13 region and ASDs (Bolton et al. 2004; Browne et al. 1997; Boyar et al. 2001; Cook et al. 1997). Lastly, the PWS individuals with a higher SCQ score $(\geq 15)$ showed a comparable level of RBS-R subscale scores with the ASD sample, except in the area of Restricted behavior, suggesting restricted behavior may be more specific to the individuals with ASDs than to PWS with comorbid ASD feature. In light of previous studies that provided evidence for linkage and association with genetic markers within the 15q11-13 region in ASDs (Cook et al. 1998; Buxbaum et al. 2002; Curran et al. 2005; Shao et al. 2003; McCauley et al. 2004; Martin et al. 2000; Philippe et al. 1999; Shao et al. 2002), the present study may support a hypothesis that at least a part of RRB manifestation in both PWS and ASDs may share a common genetic origin within the 15q11-q13 region.

In summary, we identified a very similar pattern of RRB measured on the RBS-R across different ASD cohorts, suggesting the RBS-R is robust and operates similarly across diverse participant recruitment approaches and across different age cohorts among those with ASDs. In addition, we found a comparable level of RRB between the ASD sample and PWS subgroup with higher SCQ total score, supporting a hypothesis that ASDs and PWS may share common genetic factors for RRB within the 15q11q13 region. Our study limitations include the small sample size and a wide age range especially for the PWS group, and not controlling for the potential covariates including but not limited to cognitive abilities (e.g., IQ), comorbid medical conditions and/or medications, which may influence on the RRB manifestations. Future study direction includes a replication in a larger sample set and further investigation into the genetic bases of overlapping ASD and RRB phenomenology across ASD and PWS samples.

Acknowledgements We extend our sincere gratitude to our research participants and their family members for their enthusiastic support and participation in our study. We gratefully acknowledge Susan Craft, Krista Garner, Christy Lynn, Christine Keeling, the UF Child \& Adolescent Psychiatry fellows (Drs. Isaac Isaac, Kristina Kise, Thomas Simeone, Julian Walters, Trina Webb, and Catrina Wilkins), the staff of the UF Center for Autism and Related Disabilities (CARD), and Dr. Jennifer L. Miller for their expert assistance with participants' assessment and recruitment. This work was supported in part by a 2007 NARSAD young investigator award (SJK), the 2008 PWSA (USA) Research Award (SJK), NIH R03MH083673 (SJK), NIH K23MH082883 (SJK), NIH K23MH082121 (SJ), and NIH Autism Center of Excellence P50 HD055751 (EHC).

\section{References}

APA. Diagnostic and statistical manual of mental disorders, text revision (DSM-IV-TR). 4th ed. Washington, DC: American Psychiatric Publishing; 2000.

Bishop DV, Norbury CF. Exploring the borderlands of autistic disorder and specific language impairment: a study using standardised diagnostic instruments. J Child Psychol Psychiatry. 2002;43 (7):917-29.

Bodfish JW et al. Compulsions in adults with mental retardation: prevalence, phenomenology, and comorbidity with stereotypy and self-injury. Am J Ment Retard. 1995;100(2):183-92.

Bodfish JW et al. Varieties of repetitive behavior in autism: comparisons to mental retardation. J Autism Dev Disord. 2000;30(3):237-43.

Bolton PF et al. Chromosome 15q11-13 abnormalities and other medical conditions in individuals with autism spectrum disorders. Psychiatr Genet. 2004;14(3):131-7.

Boyar FZ et al. A family with a grand-maternally derived interstitial duplication of proximal 15q. Clin Genet. 2001;60(6):421-30.

Browne $\mathrm{CE}$ et al. Inherited interstitial duplications of proximal $15 \mathrm{q}$ : genotype-phenotype correlations. Am J Hum Genet. 1997;61 (6): $1342-52$.

Butler MG et al. Behavioral differences among subjects with PraderWilli syndrome and type I or type II deletion and maternal disomy. Pediatrics. 2004;113(3 Pt 1):565-73.

Buxbaum JD et al. Association between a GABRB3 polymorphism and autism. Mol Psychiatry. 2002;7(3):311-6.

Cassidy SB, Driscoll DJ. Prader-Willi syndrome. Eur J Hum Genet. 2009;17(1):3-13.

Charman $\mathrm{T}$ et al. Efficacy of three screening instruments in the identification of autistic-spectrum disorders. $\mathrm{Br} \mathrm{J}$ Psychiatry. 2007;191:554-9.

Chandler $\mathrm{S}$ et al. Validation of the social communication questionnaire in a population cohort of children with autism spectrum disorders. J Am Acad Child Adolesc Psychiatry. 2007;46 (10):1324-32. 
Cook Jr EH et al. Autism or atypical autism in maternally but not paternally derived proximal $15 \mathrm{q}$ duplication. Am J Hum Genet. 1997;60(4):928-34.

Cook Jr EH et al. Linkage-disequilibrium mapping of autistic disorder, with 15q11-13 markers. Am J Hum Genet. 1998;62(5):1077-83.

Corsello $\mathrm{C}$ et al. Between a ROC and a hard place: decision making and making decisions about using the SCQ. J Child Psychol Psychiatry. 2007;48(9):932-40.

Curran S et al. An association analysis of microsatellite markers across the Prader-Willi/Angelman critical region on chromosome 15 (q11-13) and autism spectrum disorder. Am J Med Genet B Neuropsychiatr Genet. 2005;137(1):25-8.

Dimitropoulos A, Schultz RT. Autistic-like symptomatology in Prader-Willi Syndrome: a review of recent findings. Curr Psychiatry Rep. 2007;9(2):159-64.

Dykens EM, Leckman JF, Cassidy SB. Obsessions and compulsions in Prader-Willi syndrome. J Child Psychol Psychiatry. 1996;37 (8):995-1002.

Dykens EM, Cassidy SB, King BH. Maladaptive behavior differences in Prader-Willi syndrome due to paternal deletion versus maternal uniparental disomy. Am J Ment Retard. 1999;104(1):67-77.

Evans DW et al. Ritual, habit, and perfectionism: the prevalence and development of compulsive-like behavior in normal young children. Child Dev. 1997;68(1):58-68.

Greaves $\mathrm{N}$ et al. Repetitive and ritualistic behaviour in children with Prader-Willi syndrome and children with autism. J Intellect Disabil Res. 2006;50(Pt 2):92-100.

Horsthemke B, Buiting K. Genomic imprinting and imprinting defects in humans. Adv Genet. 2008;61:225-46.

Lam KSL. The Repetitive Behavior Scale-Revised: independent validation and the effect of subject variables. $\mathrm{PhD}$ thesis, Ohio State University, p. 129; 2004.

Lam KS, Aman MG. The Repetitive Behavior Scale-Revised: independent validation in individuals with autism spectrum disorders. J Autism Dev Disord. 2007;37(5):855-66.

Lewis M, Kim SJ. The pathophysiology of restricted repetitive behavior. J Neurodev Disord. 2009;1(2):114-32.

Lord C, Rutter M, Le Couteur A. Autism Diagnostic InterviewRevised: a revised version of a diagnostic interview for caregivers of individuals with possible pervasive developmental disorders. J Autism Dev Disord. 1994;24(5):659-85.

Lord C et al. The Autism Diagnostic Observation Schedule-Generic: a standard measure of social and communication deficits associated with the spectrum of autism. J Autism Dev Disord. 2000;30 (3):205-23
Mandy WP, Skuse DH. Research review: What is the association between the social-communication element of autism and repetitive interests, behaviours and activities? J Child Psychol Psychiatry. 2008;49(8):795-808.

Martin ER et al. Analysis of linkage disequilibrium in gammaaminobutyric acid receptor subunit genes in autistic disorder. Am J Med Genet. 2000;96(1):43-8.

McCauley JL et al. A linkage disequilibrium map of the 1-Mb $15 \mathrm{q} 12$ GABA(A) receptor subunit cluster and association to autism. Am J Med Genet B Neuropsychiatr Genet. 2004;131(1):51-9.

Milner KM et al. Prader-Willi syndrome: intellectual abilities and behavioural features by genetic subtype. J Child Psychol Psychiatry. 2005;46(10):1089-96.

Mirenda $\mathrm{P}$ et al. Validating the Repetitive Behavior Scale-revised in young children with autism spectrum disorder. J Autism Dev Disord. 2010;40(12):1521-30.

Nicholls RD, Knepper JL. Genome organization, function, and imprinting in Prader-Willi and Angelman syndromes. Annu Rev Genomics Hum Genet. 2001;2:153-75.

Philippe A et al. Genome-wide scan for autism susceptibility genes. Paris Autism Research International Sibpair Study. Hum Mol Genet. 1999;8(5):805-12.

Ronald A et al. Genetic heterogeneity between the three components of the autism spectrum: a twin study. J Am Acad Child Adolesc Psychiatry. 2006;45(6):691-9.

Rutter M, Bailey A, Lord C. Social Communication Questionnaire (SCQ). Los Angeles, CA: Western Psychological Services; 2003.

Shao $\mathrm{Y}$ et al. Genomic screen and follow-up analysis for autistic disorder. Am J Med Genet. 2002;114(1):99-105.

Shao $\mathrm{Y}$ et al. Fine mapping of autistic disorder to chromosome 15q11q13 by use of phenotypic subtypes. Am J Hum Genet. 2003;72 (3):539-48.

Silverman JM et al. Symptom domains in autism and related conditions: evidence for familiality. Am J Med Genet. 2002;114 (1):64-73.

Torrado $\mathrm{M}$, et al. Clinical-etiologic correlation in children with Prader-Willi syndrome (PWS): An interdisciplinary study. Am J Med Genet A. 2006.

Veltman MW et al. Prader-Willi syndrome-a study comparing deletion and uniparental disomy cases with reference to autism spectrum disorders. Eur Child Adolesc Psychiatry. 2004;13 (1):42-50.

Veltman MW, Craig EE, Bolton PF. Autism spectrum disorders in Prader-Willi and Angelman syndromes: a systematic review. Psychiatr Genet. 2005;15(4):243-54. 